\title{
EFECTO DEL NÚMERO DE CAMPAÑAS DE USO EN LA UNIFORMIDAD DE DISTRIBUCIÓN DE CAUDAL EN CINTAS DE RIEGO
}

\author{
Contreras París, J.I. ${ }^{1}(P)$, González Expósito, $L^{2}$., Cánovas Fernández, ${ }^{1}{ }^{1}$, Baeza Cano $R^{1}$ \\ ${ }^{1}$ Instituto de Investigación y Formación Agraria y Pesquera de Andalucía (IFAPA), Centro La \\ Mojonera, Camino San Nicolás, n¹, 04745 La Mojonera, Almería. \\ juanai.contreras@juntadeandalucia.es \\ ${ }^{2}$ Primaflor, SAT
}

\section{Resumen}

El objetivo de este trabajo fue estudiar el efecto del número de campañas de uso sobre la uniformidad de distribución de caudal en una cinta de riego con emisores con compensación de caudal, con el fin de determinar su vida útil. El experimento se ha desarrollado en el laboratorio de materiales de riego localizado, situado en las instalaciones del Centro IFAPA La Mojonera en La Cañada (Almería). El dispositivo experimental instalado consistió en un banco de pruebas de cintas de riego de $40 \mathrm{~m}$ de longitud. Se evaluó el comportamiento de un modelo de cinta de riego con emisores autocompensantes de uso frecuente en el cultivo de lechuga (DripNet PC ${ }^{\mathrm{TM}}$ de Netafim). Se estableció un diseño experimental con siete tratamientos y tres repeticiones donde la unidad experimental básica fue un lateral de cinta de riego con 200 salidas. Los tratamientos estudiados fueron los números de ciclos de cultivo de lechuga (1, 2, 3, 5, 6, 8 y 10 usos) y las repeticiones las presiones de trabajo $(0.10,0.15,0.20 \mathrm{MPa})$. Los resultados han permitido establecer una función preliminar que define la relación entre la uniformidad de riego y el número de usos para el modelo de cinta estudiada. La uniformidad de distribución de caudal fue superior al $85 \%$ hasta cuando la cinta fue utilizada en 8 ciclos de lechuga, si bien desciende en el caso de 10 usos a un $53 \%$. Por tanto, se podría concluir que la vida útil de una cinta de riego con emisores autocompensantes DripNet PC ${ }^{\mathrm{TM}}$ es de ocho usos para las condiciones de cultivo estudiadas (cultivo de lechuga con instalación subterránea de las cintas), si bien, sería conveniente repetir el ensayo con diferentes tratamientos de mantenimiento para sacar unas conclusiones definitivas.

\section{1.- Introducción y Objetivos}

Las cintas de riego son un material muy utilizado en los sistemas de producción de cultivos hortícolas, tanto en invernadero como al aire libre. Su instalación puede ser subterránea o superficial. En Andalucía se ha generalizado su empleo en cultivos como la fresa y la lechuga. Entre las principales ventajas que presentan, con respecto a los emisores convencionales de riego localizado, se encuentran el menor coste y la mayor ligereza. Esta última característica facilita las labores de instalación y retirada del sistema de riego de las parcelas de cultivo para el acondicionamiento del terreno al inicio de los ciclos productivos.

En instalaciones de riego localizado es posible encontrar una elevada uniformidad de distribución cuando se evalúa la instalación en carga, observándose descensos en la misma conforme aumenta la vida de los emisores (Baeza et al., 2010, 2014). Las investigaciones más recientes sobre la uniformidad de riego en cintas de riego por goteo se centran en el 
efecto de la presión de funcionamiento (Safi et al., 2007; Poh et al., 2011; Wang et al., 2012), la longitud de la cinta (Safi et al., 2007; Poh et al., 2011; Wang et al., 2012; Provenzano et al., 2014; Baiamonte et al., 2015), la profundidad de colocación de cinta en riego subterráneo (Patel y Raiput, 2007) y la pendiente del terreno (Wang et al., 2012).

En principio, las cintas de riego, por la baja durabilidad del material están pensadas para una o dos campañas de trabajo, aunque este aspecto está muy influenciado por las labores de mantenimiento realizadas (tratamientos de acidificación, limpieza...) que permiten alargar su vida útil.

Actualmente, las empresas fabricantes ofertan cintas de riego con emisores integrados de las mismas características que los utilizados en los sistemas convencionales de riego localizado, que permitirían una mayor vida útil de las mismas, además de ofrecer la posibilidad de integrar emisores con compensación de caudal que hacen posible trabajar en terrenos con elevada pendiente sin descenso en la uniformidad de distribución de caudal durante el riego. Todos estos nuevos materiales tienen un mayor coste y los usuarios están intentando aumentar el número de usos para mejorar su amortización. Sin embargo, no existen estudios que indiquen la vida útil de este tipo de materiales.

El objetivo de este trabajo fue estudiar el efecto del número de usos sobre la uniformidad de distribución de caudal en una cinta de riego con emisores con compensación de caudal, con el fin de determinar su vida útil.

\section{2.- Materiales y Métodos}

El experimento se ha desarrollado en el laboratorio de materiales de riego localizado, situado en las instalaciones del Centro IFAPA La Mojonera en La Cañada (Almería). EI dispositivo experimental instalado consistió en un banco de pruebas de cintas de riego de 40 $\mathrm{m}$ de longitud situado a nivel. Se evaluó el comportamiento de un modelo de cinta de riego con emisores autocompensantes de uso frecuente en el cultivo de lechuga (DripNet PC ${ }^{\text {TM }}$ de Netafim). Se estableció un diseño experimental con siete tratamientos y tres repeticiones donde la unidad experimental básica fue un lateral de cinta de riego con 200 salidas. Los tratamientos estudiados fueron los números de ciclos de cultivo de lechuga $(1,2,3,5,6,8$ y 10 usos) y las repeticiones las presiones de trabajo $(0.10,0.15,0.20 \mathrm{MPa})$. Las cintas de riego fueron sometidas a pulsos de riego de 15 minutos y se midió el volumen recogido en 28 emisores durante todo el pulso de riego, incluyendo la descarga de la cinta. Se determinó el coeficiente de uniformidad de caudal (CUC) mediante el método propuesto por Merriam \& Keller (1978) (Ecuación 1):

$\operatorname{CUC}=\left(\mathrm{q}_{25 \%} / \mathrm{q}_{\mathrm{m}}\right) \times 100$

(Ecuación 1)

donde:

$\mathrm{q}_{25 \%}=$ caudal medio del $25 \%$ de emisores con menor descarga $\left(L h^{-1}\right)$.

$\mathrm{q}_{\mathrm{m}}=$ caudal medio de todos los emisores evaluados $\left(\mathrm{L} \mathrm{h}^{-1}\right)$.

Los valores de CUC fueron clasificados según by Merriam y Keller (1978) (Tabla 1). 
Tabla 1. Clasificación del coeficiente de uniformidad de caudal (CUC) por Merriam y Keller (1978).

\begin{tabular}{cc}
\hline CUC & Clasificación \\
\hline$>95 \%$ & Excelente \\
$85 \%-95 \%$ & Bueno \\
$80 \%-85 \%$ & Aceptable \\
$70 \%-80 \%$ & Pobre \\
$<70 \%$ & Inaceptable \\
\hline
\end{tabular}

Antes de realizar las medidas en el banco de pruebas, para cada cinta correspondiente al número de usos estudiados, se determinó el rendimiento hidráulico del emisor mediante una función empírica que relaciona el caudal emitido por el emisor en función de la presión de funcionamiento (curva de gasto del emisor) (Keller y Karmeli, 1974; ASABE, 2005) (Ecuación 2):

$$
Q=k P^{x}
$$

donde:

$Q$ es el caudal de descarga del emisor $\left(L^{-1}\right)$

$\mathrm{k}$ es la constante del emisor

$\mathrm{P}$ presión de trabajo (MPa)

$x$ es el exponente de descarga

El banco de ensayo utilizado para la determinación las curvas de gasto de los emisores se diseñó según la norma UNE 68-075-86 (Figura 1), evaluando veinticinco emisores. 


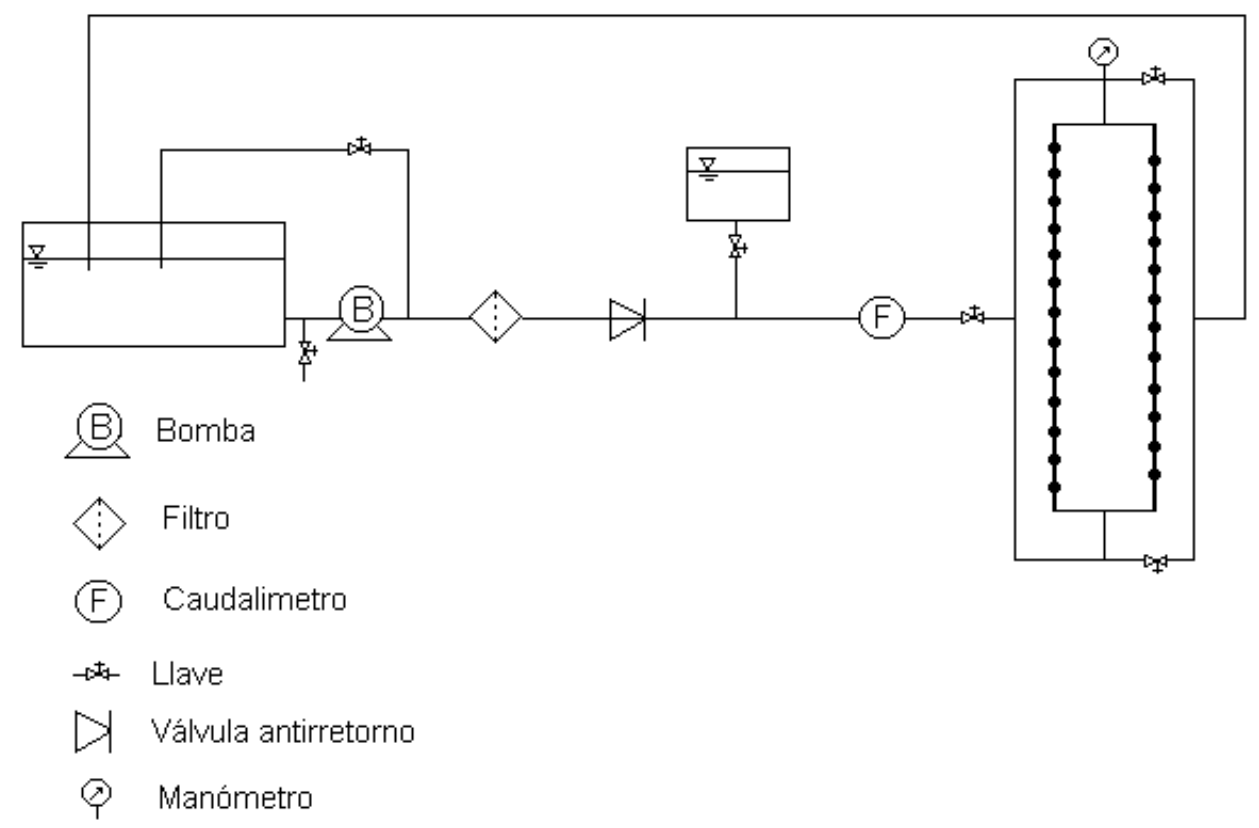

Figura 1. Esquema del banco de pruebas de emisores de riego localizado.

Los resultados han sido analizados estadísticamente con el software STATGRAPHICS Plus (versión 5.1). Las ecuaciones de gasto del emisor se realizaron mediante una regresión lineal con un ajuste multiplicativo. Se realizó un análisis ANOVA para identificar el efecto de los tratamientos estudiados. Cuando el análisis estadístico reveló diferencias significativas entre tratamientos se aplicó un test de comparación de media (LSD; mínima diferencia significativa) con $p \leq 0.05$.

\section{3.- Resultados y conclusiones}

Los parámetros que definen las curvas de gasto de los emisores en función de las campañas de uso se muestran en la tabla 2 . El número de campañas de uso de la cinta de riego no afectó la compensación de caudal, ya que el exponente de descarga de los emisores no se ha visto modificado por el número de usos, en todos los caso se ha situado muy próximo a cero.

Tabla 2. Valores of $k$ y $x$ para los emisores analizados.

\begin{tabular}{llll}
\hline$N^{\circ}$ de Campañas de uso & $k$ & $\mathrm{x}$ & Formula de gasto \\
\hline 1 & 6.55 & 0.024 & $\mathrm{Q}\left(\mathrm{Lh}^{-1}\right)=6.54869^{*} \mathrm{P}(\mathrm{MPa})^{0.0237929}$ \\
2 & 6.41 & 0.012 & $\mathrm{Q}\left(\mathrm{Lh}^{-1}\right)=6.40643^{*} \mathrm{P}(\mathrm{MPa})^{0.011943}$ \\
3 & 6.60 & -0.023 & $\mathrm{Q}\left(\mathrm{Lh}^{-1}\right)=6.60234^{*} \mathrm{P}(\mathrm{MPa})^{-0.0231667}$ \\
5 & 6.54 & -0.002 & $\mathrm{Q}\left(\mathrm{Lh}^{-1}\right)=6.54029^{*} \mathrm{P}(\mathrm{MPa})^{-0.00168198}$ \\
6 & 6.54 & -0.001 & $\mathrm{Q}\left(\mathrm{Lh}^{-1}\right)=6.54002^{*} \mathrm{P}(\mathrm{MPa})^{-0.00138868}$ \\
8 & 6.45 & 0.050 & $\mathrm{Q}\left(\mathrm{Lh}^{-1}\right)=6.44573^{*} \mathrm{P}(\mathrm{MPa})^{0.0500578}$ \\
10 & 6.18 & -0.009 & $\mathrm{Q}\left(\mathrm{Lh}^{-1}\right)=6.17649^{*} \mathrm{P}(\mathrm{MPa})^{-0.00925245}$ \\
\hline
\end{tabular}


Sin embargo, a pesar de que el caudal medio ha sido similar en todos los usos, tal y como muestra la escasa modificación observada en la fórmula de gasto (Tabla 2), ha aumentado la variabilidad de los caudales emitidos, viéndose afectada la uniformidad de riego (CUC). Esta variabilidad está asociada a las alteraciones de funcionamiento que se producen en las membranas de los emisores autocompensantes cuando se depositan sedimentos en ellas. Los sedimentos pueden ocasionar tanto obturaciones, como el efecto contrario, incrementos de caudal.

El número de usos ha tenido un efecto estadísticamente significativo en la uniformidad de riego, sin embargo, la presión de trabajo no ha afectado significativamente la uniformidad (Tabla 3).

Tabla 3. Nivel de significación de los factores estudiados sobre el coeficiente de uniformidad de caudal (CUC).

\begin{tabular}{|l|c|}
\hline Factor & CUC (\%) \\
\hline Número de campañas de uso & ${ }^{*}$ \\
\hline Presión de trabajo & ns \\
\hline ns, no significativo. \\
*Significativo para $P<0.05$.
\end{tabular}

Los CUC medios obtenidos en los emisores, en función del número de campañas de riego, se muestra en la tabla 4. Hasta la quinta campaña de riego, incluida ésta, no se mostraron diferencias significativas en la uniformidad de riego, presentando valores buenos de uniformidad según la clasificación de Merriam y Keller (1978). Sin embargo, en la sexta campaña de uso se registró un descenso de la uniformidad, situándose en valores aceptables, descenso que se acentuó en la décima campaña de uso, registrándose una uniformidad inaceptable (53\%).

Tabla 4. Efecto del número de campañas de uso en el coeficiente de uniformidad de caudal (CUC).

\begin{tabular}{|c|c|}
\hline$N^{\circ}$ de campañas de uso & CUC (\%) \\
\hline 1 & $92.48 \mathrm{a}$ \\
\hline 2 & $89.86 \mathrm{ab}$ \\
\hline 3 & $92.46 \mathrm{a}$ \\
\hline 5 & $95.54 \mathrm{a}$ \\
\hline 6 & $78.17 \mathrm{c}$ \\
\hline 8 & $86.96 \mathrm{~b}$ \\
\hline 10 & $52.78 \mathrm{~d}$ \\
\hline \multicolumn{2}{|c|}{} \\
Letras diferentes en la misma columna indican diferencias \\
estadísticamente significativa para P<0,05
\end{tabular}

Los resultados permitieron establecer una función preliminar que define la relación entre la uniformidad de riego y el número de usos para el modelo de cinta estudiada. Según la curva descrita, la uniformidad de distribución de caudal se sitúa en torno al $90 \%$ hasta las cinco campañas de uso, a partir de la cual desciende. Si bien la uniformidad puede ser superior al $85 \%$ hasta cuando la cinta es utilizada en 8 ciclos de lechuga, descendiendo drásticamente en el caso de 10 usos a un 53\% (Figura 2). 


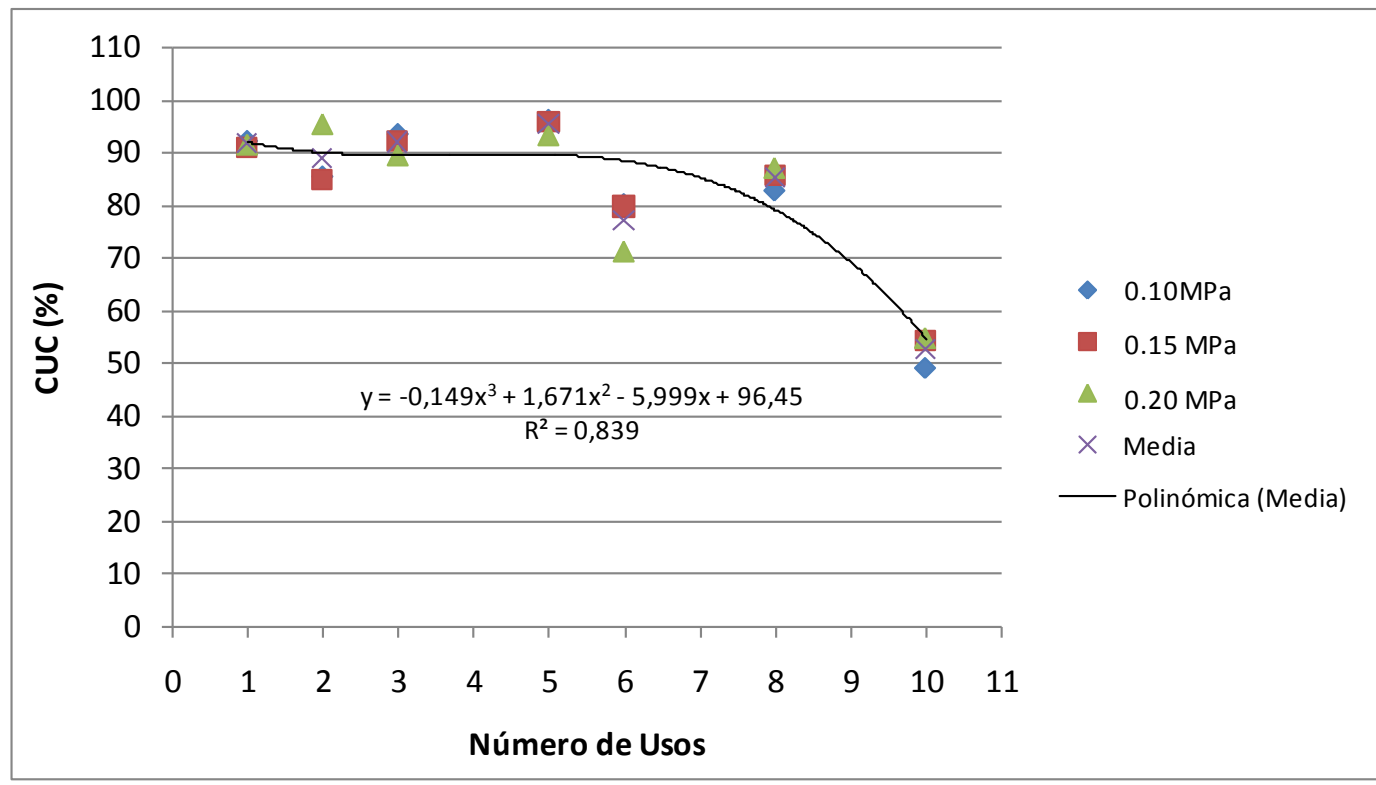

Figura 2. Efecto del número de campañas de uso sobre el coeficiente de uniformidad de caudal (CUC).

Se ha observado que el número de fugas de la cinta puede ser elevado dependiendo del manejo de la misma, al igual que el número de goteros totalmente obturados que no está relacionado con el uso de la cinta ni con la presión de trabajo, sino con el manejo, ya que las obturaciones eran ocasionadas principalmente por presencia de arcilla en el emisor. Esta arcilla se introduce principalmente en las operaciones de instalación y desinstalación de la cinta.

Con los resultados obtenidos se podría concluir que la vida útil de una cinta de riego con emisores autocompensantes DripNet $\mathrm{PC}^{\mathrm{TM}}$ es de ocho usos para las condiciones de cultivo estudiadas (cultivo de lechuga con instalación de cintas enterradas), siempre y cuando se realice unas labores adecuadas de mantenimiento que minimicen las fugas si bien, sería conveniente repetir el ensayo con diferentes tratamientos de mantenimiento para sacar unas conclusiones definitivas.

\section{4.-Agradecimientos}

El trabajo ha sido cofinanciado por Fondos Europeos (FSE y FEDER) y la Consejería de Agricultura y Pesca (IFAPA Junta de Andalucía) mediante el Proyecto Transforma Conecta SAR (PP.TRA.TRA201300.10).

\section{5.-Bibliografía}

Al-Amoud, A.I., Mattar, M.A. \& Ateia, M.I. (2014). Impact of water temperature and structural parameters on the hydraulic labyrinth-channel emitter performance. Span. J. Agric. Res. 12(3): 580-593.

ASABE (2005). EP-458: Field evaluation of microirrigation systems. ASABE, St. Joseph, USA.

Baeza Cano, R., Gavilán Zafra, P., Del Castillo Lupiáñez, N., Berenguel, P. \& López Segura, J.G. (2010). Programa de evaluación y asesoramiento en instalaciones de riego en 
invernadero con uso de dos fuentes distintas de agua: subterránea y regenerada. XXVIII Congreso Nacional de Riegos. León 15-17 Junio de 2010.

Baeza, R., Gavilán P. \& Contreras, J.I. (2014). Influencia de la pendiente del terreno en la uniformidad de distribución de caudal en cintas de riego localizado. XXXII Congreso Nacional de Riegos. AERYD. Madrid, 10-12 junio de 2014.

Baiamonte, G., Provenzano, G. \& Rallo, G. (2015). Analytical Approach Determining the Optimal Length of Paired Drip Laterals in Uniformly Sloped Fields.J. Irrig. Drain Eng.141(1) 04014042. Online publication date: 1-Jan-2015.

Gavilán, P., Lozano, D., Ruiz, N. \& Molina, F. (2014). El riego de la fresa en el entorno de Doñana. Evapotranspiración, coeficientes de cultivo y eficiencia del riego. XXXII Congreso Nacional de Riegos. AERYD. Madrid, 10-12 junio de 2014.

Keller, J. \& Karmeli, D. (1974). Trickle irrigation design parameters. Transaccion of the ASAE.

Lozano, D., Ruiz, N. \& Gavilán, P. (2014). Evaluación de la uniformidad de distribución de cintas de riego en condiciones de campo en una producción comercial de fresa en Almonte. XXXII Congreso Nacional de Riegos. AERYD. Madrid, 10-12 junio de 2014.

Merriam, J.L. \& Keller J. (1978). Farm irrigation system evaluation: a guide for management. UTAH State University. Logan, Utah, USA.

Patel, N. \& Rajput, T. B. S. (2007). Effect of drip tape placement depth and irrigation level on yield of potato. Agric. Water Manage. 88(1):209-223.

Poh, B.L., Gazula, A., Simonne, E.H., Hochmuth, R.C. \& Alligood, M.R. (2011). Use of Reduced Irrigation Operating Pressure in Irrigation Scheduling. II. Effect of Reduced Irrigation System Operating Pressure on Drip-tape Flow Rate, Water Application Uniformity, and Soil Wetting Pattern on a Sandy Soil. HortTechnology 21(1); 22-29.

Provenzano, G., Di Dio, P. \& Leone, R. (2014). Assessing a Local Losses Evaluation Procedure for Low-Pressure Lay-Flat Drip Laterals. J. Irrig. Drain Eng. 140(6): 04014017.

Safi, B., Neyshabouri, M.R., Nazemi, A.H., Massiha, S. \& Mirlatifi, S. M. (2007). Water application uniformity of a subsurface drip irrigation system at various operating pressures and tape lengths. Turk J Agric For 31(5):275-285.

UNE 68-075-86. (1986). Material de riego. Emisores. Requisitos generales y métodos de ensayo. Norma Española.

Wang, X., Cui, C.N., Shalamu, A. \& Shen, X.M. (2012). Field Experiment and Analysis of Irrigation Uniformity of Different Drip Irrigation Tapes. Water Saving Irrigation 8: 005. 